\title{
Risks of Misinterpretation in the Evaluation of the Effect of Fruit-Based Drinks in Postprandial Studies
}

\author{
Ilaria Peluso ${ }^{1}$ and Maura Palmery ${ }^{2}$ \\ ${ }^{1}$ Food and Nutrition Center of the Agricultural Research Council (CRA-NUT), Via Ardeatina 546, 00178 Rome, Italy \\ ${ }^{2}$ Department of Physiology and Pharmacology "V. Erspamer", "Sapienza" University of Rome, \\ Piazzale Aldo Moro 5, 00185 Rome, Italy \\ Correspondence should be addressed to Ilaria Peluso; i.peluso@tiscali.it
}

Received 27 October 2014; Accepted 14 December 2014; Published 25 December 2014

Academic Editor: Gianfranco D. Alpini

Copyright (c) 2014 I. Peluso and M. Palmery. This is an open access article distributed under the Creative Commons Attribution License, which permits unrestricted use, distribution, and reproduction in any medium, provided the original work is properly cited.

\begin{abstract}
It has been suggested that some fruit-based drinks (FBD) may delay the onset of postprandial stress, which is involved in the pathogenesis of many diseases. The majority of the studies, which have investigated the effects of FBD on postprandial stress, involved a placebo that was a drink with the same content in sugars or carbohydrates of the FBD, but without the bioactive antioxidant compounds. These studies were aimed more at evaluating the effect of the antioxidants rather than the effect of the FBD as a whole. Only 4 studies compared the effect of FBD with water as control and did not support the hypothesis that FBD could inhibit postprandial dysmetabolism, as well as the studies that compared the effect of orange juice and cola. Overall, the results suggest a complex relationship between postprandial dysmetabolism, inflammation, and oxidative stress. Furthermore, the inflammatory and oxidative stress markers need further analytical validation and normal ranges should be established in order to reach a firm conclusion. Finally, caution should be taken in the interpretation of the effect of FBD in postprandial studies and the reviewed results suggest that dietary recommendations should aim to limit rather than increase sugar-sweetened beverages consumption.
\end{abstract}

\section{Introduction}

The frequent consumption of high fat (HFM), high carbohydrates (HCM), or mixed high fat and high carbohydrates (HFHCM) meals is associated with an increased risk of diabetes and cardiovascular disease (CVD) [1-3]. This association seems attributable to the onset of a condition defined as "low-grade chronic inflammation," which is mediated by inflammatory cytokines and oxidative stress [4]. In particular, some cells associated with the innate immune response respond to the acute postprandial lipid increase with the oxidative burst [4]. It has been suggested that some fruitbased drinks (FBD) may delay the onset of postprandial stress $[2,3]$.

However, although FBD contain many flavonoids that act as antioxidants $[3,5]$, the human studies, which have investigated the effect of these polyphenols on immune function, have given contrasting results $[3,6]$. Moreover, it has been suggested to avoid soft drinks containing sugars, including fruit juices that contain a lot of fructose, in the nutritional management of nonalcoholic fatty liver disease (NAFLD) [7], inflammatory bowel disease (IBD), and irritable bowel syndrome (IBS) [8].

We aim to review the results of the studies that have investigated the effects of FBD by using the postprandial stress as acute model of inflammation, taking into account the control used for the comparison.

\section{The Postprandial Stress as Acute Model of Inflammation}

It has been suggested that the dynamic models of acute inflammation may provide much more sensitive and meaningful information of the health state of an individual, with respect to the assessment of fasting inflammatory and metabolic markers [1]. The models of acute inflammation can 
be classified on the basis of the dominant event that triggers the inflammatory response and may therefore be divided into metabolic overload (oral glucose or lipids), stress from infection (injection of the endotoxin lipopolysaccharide, LPS), hypersensitivity responses to vaccines, and tissue damage (strenuous exercise or exposure to UV light) [1].

In Western society, the two prevalent origins of oxidative stress are the ingestion of HFM and the performance of strenuous exercise. However, in exercise-trained men, the magnitude of oxidative stress following HFM is significantly greater than that elicited by either aerobic or anaerobic exercise [24]. On the other hand, exercise improves postprandial glucose metabolism $[25,26]$ and the activation of leukocytes induced by lipaemia $[27,28]$. However, exercise does not influence postprandial triglycerides (TG) and oxidative stress in obese prediabetic women [29]. In fact, obesity and type 2 diabetes (T2D) are considered important factors in the extent of postprandial stress, which is greater in subjects with the metabolic syndrome [1]. The extent of the postprandial inflammatory response seems to be correlated with the degree of insulin resistance [30,31]. We have recently suggested that repeated hyperinsulinemia could be induced to inhibit the postprandial inflammation [4]. On the other hand, the increased activation of leukocyte in T2D may be due to the fact that metabolic diseases and obesity are associated with an increased intestinal permeability and an increased translocation of LPS from the lumen to the mucosa $[1,4$, 32]. In fact, the most accepted mechanism of the postprandial activation of leukocyte is the phenomenon known as metabolic endotoxemia $[4,32]$. The endotoxemia may be caused by direct spread of the LPS from the intestinal lumen, due to increased intestinal paracellular permeability, or through the absorption by enterocytes during the process of secretion of chylomicrons $[4,32]$. HFHCM induces an increase of LPS and inflammatory cytokines [4]. The primary role of lipids, in the postprandial endotoxemia, is supported by the evidence that while HFM increased the levels of tumor necrosis factor-alpha (TNF- $\alpha$ ), interleukin 1-beta (IL$1 \beta)$, LPS, and Toll-like receptor-4 (TLR4), the ingestion of glucose increased only TNF- $\alpha$ and IL-1 $\beta$ [33]. TLR4, along with the LPS receptor (CD14), is involved in the induction of the oxidative burst and may be involved also in the activation of monocytes by urate crystals that form in the case of hyperuricemia [4]. Therefore, the increase in uric acid (UA) after HFM could enhance the innate immune response and the production of reactive oxygen species (ROS) [4]. In this context, it has been suggested that dietary fructose may increase plasma UA [34] and it is well known that fructose overfeeding increases fasting and postprandial plasma TG concentrations, which are related to the stimulation of hepatic de novo lipogenesis and to a decreased clearance of TGrich lipoproteins [35-39]. The dyslipidaemic effect of dietary fructose occurs also in healthy children and is higher in those with NAFLD [40]. On the contrary, it has been recently reported that moderate amounts of high fructose or high glucose sweetened beverages for 2 weeks did not have differential effects on postprandial TG in physically active adolescents [41]. These results could be due to the lowering effect of exercise on the percentage of CD14 positive cells, the TLR4 expression, the LPS-stimulated production of TNF$\alpha$, and the increases in ROS, all induced by postprandial lipaemia $[27,28]$. The role of CD14, TLR4, LPS, and TNF$\alpha$ in the development of NAFLD [42-44] could account also for the protective effect of the physical activity in this disease.

\section{The Effect of FBD on Postprandial Stress}

Some studies have investigated the effects of FBD on postprandial stress (Tables 1 and 2) [9-23].

The postprandial glucose increase is characterized by a peak at around 30 minutes after the meal; then, glucose returns to baseline approximately two hours after the meal (in healthy subjects), consistent with the insulin response. On the other hand, the majority of the studies that evaluated the effect of HFM recorded a peak of TG 3-5 hours after the meal. Increases in inflammatory cytokines can be found with a peak between 3 and 6 hours after the meal and TNF- $\alpha$ in particular has a significant role in the oxidative burst induced by postprandial lipaemia [4]. Therefore, in order to follow the inflammatory response, markers of inflammation and oxidative stress were analyzed, in particular, the cytokines, the oxidative burst, the products of lipoperoxidation, and the nonenzymatic antioxidant capacity (NEAC) (Tables 1 and 2).

In the studies reported in the literature (Tables 1 and 2), the quality and the quantity of the ingested fats vary widely and often the effect of the simultaneous consumption of sugars and lipids was analyzed. The majority of the studies involved a placebo that was a drink with the same sugars or carbohydrates $(\mathrm{CHO})$ content of the $\mathrm{FBD}$, but without the bioactive antioxidant compounds (polyphenols and vitamin C). These studies were aimed more at evaluating the effect of antioxidants rather than the effect of FBD as a whole (Table 1) [9-19]. Furthermore, Snyder et al. [19] aimed to evaluate the effect of polyphenols contained in navel orange juice; therefore, they used a placebo matched in energy, sucrose, fructose, glucose, and ascorbic acid (Table 1). On the other hand, only 4 studies evaluated the effect of FBD with water as control (Table 2) [20-23].

3.1. Studies with Placebo Matched in Carbohydrates. Eleven studies (14 interventions) [9-19] investigated the effect of FBD by using a placebo matched in $\mathrm{CHO}$ (Table 1). Only in two interventions $[9,11]$ the subjects received a HFM with the placebo beverage at the end of a period of 6 weeks of supplementation with either a strawberry or a placebo beverage. The same HFM, HCM, or HFHCM was used in each study and sometimes the same meal was used in different studies (Table 1). Three studies [9-11] used a test meal composed of bagel $(110 \mathrm{~g})$, cream cheese $(14 \mathrm{~g})$, margarine $(5 \mathrm{~g})$, hardboiled egg (50 g), and whole milk (240 g). Three studies [1618 ] used a meal composed of fried potatoes $(212 \mathrm{~g})$, fried eggs $(108 \mathrm{~g})$, cheese $(90 \mathrm{~g})$, and white bread $(90 \mathrm{~g})$. Two studies $[13,15]$ used a meal consisting of one EggMcMuffin, one Sausage McMuffin, and two hash brown patties. The other three studies used $200 \mathrm{~g}$ of cream and $75 \mathrm{~g}$ of sucrose [12], high-oleic sunflower oil baked into a muffin [14], or $28 \mathrm{~g}$ of Kellogg's Corn Flakes and $118 \mathrm{~mL}$ of 2\% milk [19]. 


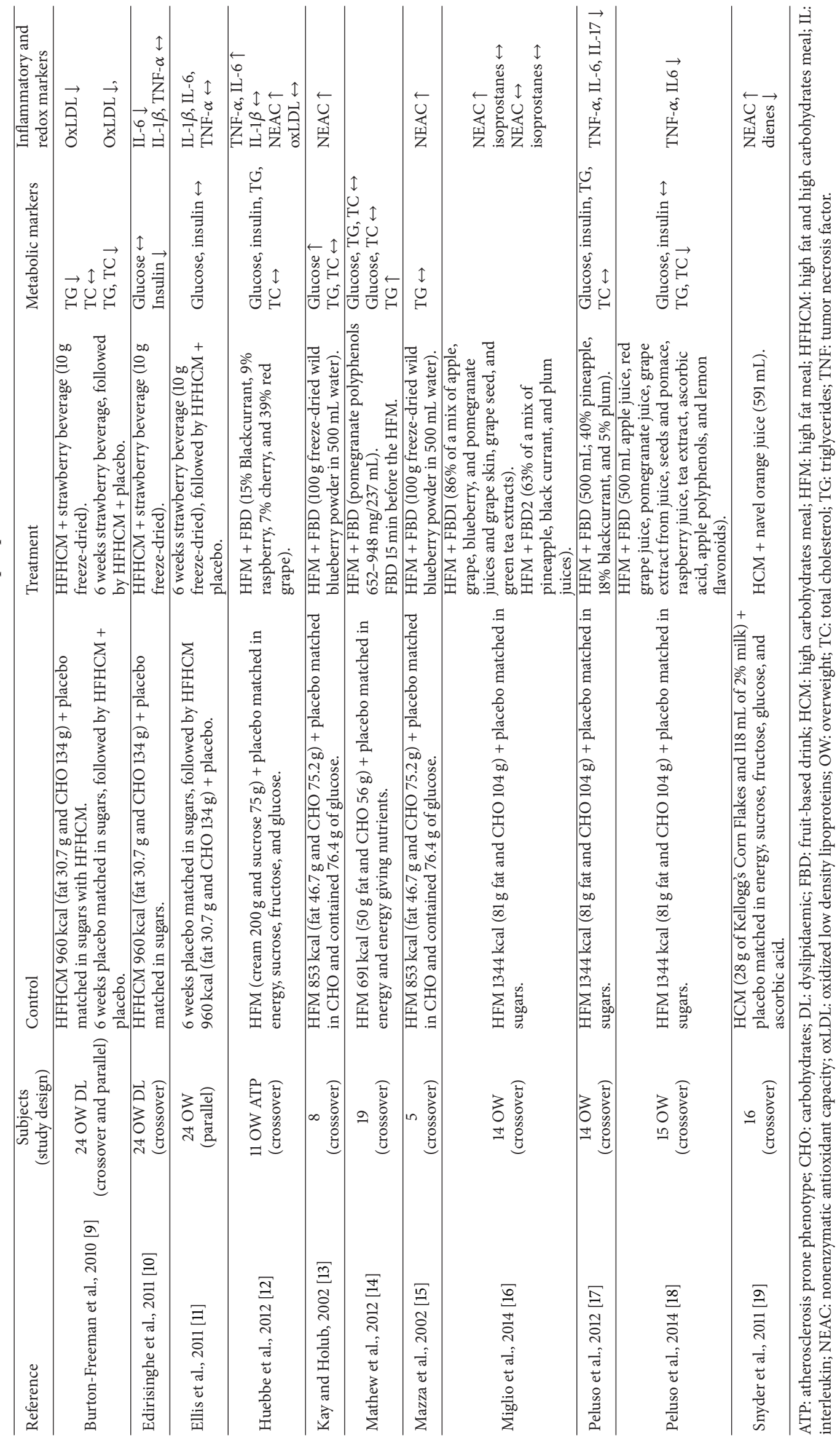




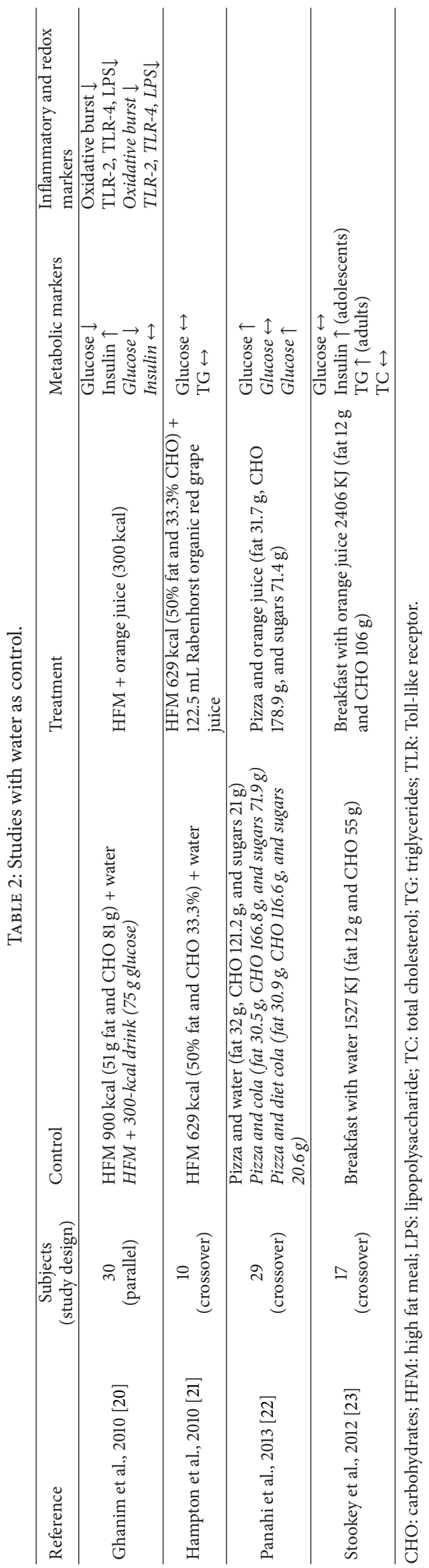


The majority of the interventions did not affect postprandial TG (55.5\%), total cholesterol (TC, 75\%), insulin (80\%), and glucose $(87.5 \%)$. Only 3 interventions reported decreased levels of TG in overweight (OW) subjects, of which two involved dyslipidaemic individuals [9] and one involved a meal containing $81 \mathrm{~g}$ of fat and a FBD containing not only fruit juices and extracts (Table 1) but also a tea extract [18]. In this context, it is known that tea catechins reduce TG absorption [45] and that $80 \mathrm{~g}$ of lipids induced a marked effect on postprandial TG [3]. On the other hand, Mathew et al. [14] observed different effects on TG and glucose when the FBD was consumed during (DUR) or 15 min before (PRE) the HFM. The drink contained a pomegranate extract and caused a greater increase in postprandial TG after PRE compared to control, whereas a mean increase of $0.44 \mathrm{mmol} / \mathrm{L}$ in plasma glucose concentrations was found following DUR compared to the mean decreases of $-0.25 \mathrm{mmol} / \mathrm{L}$ and $-0.26 \mathrm{mmol} / \mathrm{L}$ following control and PRE, respectively.

Only Edirisinghe et al. [10] reported that postprandial plasma insulin concentrations in OW dyslipidaemic individuals were significantly lower, compared to the placebo beverage, when a strawberry beverage was consumed with the test meal. However, plasma glucose concentrations were not different between the treatments. Furthermore, the placebo used in the studies of this group [9-11] was matched in sugars (51.6) but had a different content of white granulated sugar (10 g) compared to the strawberry beverage ( $3 \mathrm{~g})$.

Kay and Holub [13] reported that serum glucose concentrations were significantly higher in the blueberry group at 3 and $4 \mathrm{~h}$ than in control, in accordance with the delayed onset of postprandial glucose after fructose ingestion [46].

In dyslipidaemic subjects, the postprandial TG levels were significantly lower, compared with placebo, when a strawberry beverage was consumed with HFM or for 6 weeks before the test meal plus placebo [9]. The strawberry beverage decreased the oxidized low density lipoproteins (oxLDL) both when it was consumed with the HFM and when it was consumed for 6 weeks before the HFM challenge. On the other hand, the decrease in IL-6 was observed only when strawberry beverage was consumed during the HFM and no effect was observed on TNF- $\alpha$ and IL- $1 \beta$ (Table 1 ).

Concerning the effect of FBD on the postprandial increase of cytokines, TNF- $\alpha$ and IL- 6 decreased, respectively, in $40 \%$ and $60 \%$ of the interventions (Table 1).

On the contrary, Huebbe et al. [12] in OW atherosclerosisprone phenotype subjects, using a FBD and a placebo beverage identical in energy, sucrose, fructose, and glucose, adjusted for dietary fibre content and different in ascorbic acid $(122 \mathrm{mg} / 250 \mathrm{~g})$ and total polyphenol content $(617 \mathrm{mg}$ gallic acid equivalents $/ 250 \mathrm{~g}$ ), observed an increase in the postprandial IL-6 (plasma values) and TNF- $\alpha$ (ex vivo), despite the increase of NEAC and ascorbic acid and the nonsignificant effect on IL-1 $\beta$ (ex vivo), oxLDL, glucose, insulin, TG, and TC.

The lipoperoxidation markers decreased in $50 \%$ of the interventions, whereas an increase in NEAC was observed in $83.3 \%$ of the interventions. Despite the fact that the consumption of HFHCM has been associated with oxidative stress and with a decline in antioxidant defences in plasma, increases in NEAC have been reported following HFM [13, 16]. The increase in TG over time, but not serum glucose, was positively correlated with the increase in NEAC [13]. In OW subjects, after the consumption of a HFM, increased UA levels were documented, in conjunction with an increase of TG, TNF- $\alpha$, and IL-6 [47]. The increase in endogenous antioxidants after HFM could account for the increase of NEAC [47]. Despite the ability of fructose to increase UA, two FBD with a different antioxidant capacity and with a different fructose content (FBD1: total radical-trapping antioxidant parameter (TRAP) $16.4 \mathrm{mM}$, ferric reducing antioxidant potential (FRAP) $27.6 \mathrm{mM}$, and fructose $57 \mathrm{~g} / \mathrm{L}$; FBD2: TRAP 9.7 mM, FRAP $13.6 \mathrm{mM}$, and fructose $29 \mathrm{~g} / \mathrm{L})$ ) showed the same inhibitory effect on UA, which reached a peak $2 \mathrm{~h}$ after HFM [16]. On the other hand, only the FBD1 intervention significantly increased the urinary and plasma NEAC.

Overall, the studies that have investigated the effect of the antioxidants contained in FBD on postprandial stress (Table 1) have given contrasting results due to the differences in fat and $\mathrm{CHO}$ content of the meal and in glucose and fructose content of the placebo beverage.

3.2. Studies That Evaluated the Effect of Fruit Juice. Studies that evaluated the effects of fruit juices on HFM or mixed meals with water as control in healthy subjects are described in Table 2. Two of these studies compared also the effect of orange juice and drinks matched in sugars (placebo or cola) on postprandial glucose. Total calories from the intake of beverage with pizza were higher following the caloric beverages (orange juice and regular cola) compared to either water or diet cola [22]. Authors found higher blood glucose after the meals with orange juice and regular cola compared to diet cola and water [22]. Also Sullivan and Scott [48], in noninsulin-dependent diabetes mellitus patients, found no difference in the postprandial serum glucose after Coke or orange juice consumption. Cola and orange juice were the beverages of the two isocaloric meals ( $820 \mathrm{kcal}, 41 \%$ fat, and $42 \% \mathrm{CHO}$ ) compared by Ramel et al. [49]. The conventional fast-food meal was a hamburger meal (hamburger, bacon, and cola drink) and the unconventional fast food was a salmon-burger meal (fiber-rich sourdough rye bread, salad with vinegar, and orange juice). The postprandial increases in glucose and insulin were $44 \%$ lower after the unconventional meal, but this effect could be due to the fiber content of this meal.

On the other hand, despite glucose concentrations rise after HFHCM with water and glucose, but not with orange juice, the increase in insulin concentrations was significantly higher with glucose or orange juice compared to water [20]. Accordingly Stookey et al. [23], in a crossover design on 7 adolescents and 10 adults, observed that plasma insulin concentrations were significantly higher after breakfast with orange juice. Furthermore, serum TC and TG did not differ significantly across study days in the adolescents, but TG levels were significantly higher after breakfast with orange juice in adults. On the other hand, Hampton et al. [21] observed a nonsignificant increase in glucose after a meal consisting of vegetable lasagne and cake accompanied with Rabenhorst 
organic red grape juice and no effect on postprandial TG. The contrasting results on TG of these studies [21, 23] could be due to the different content of fat of the two meals (Table 2). In fact, it is known that the amount of lipid required to cause a significant increase in the levels of TG is of the order of 30$50 \mathrm{~g}$, with low response to 5-15 g [3]. Probably the increase in TG induced by fructose could be appreciated only with low fat meals (LFM).

In this context, 2 consecutive studies were conducted on coronary artery disease (CAD) [50] or healthy subjects [51]. Bae et al. compared the effect of a HFM ( $803 \mathrm{kcal})$ which consisted of $110 \mathrm{~g}$ rice, $100 \mathrm{~g}$ Korean barbecue, $20 \mathrm{~g}$ egg, $200 \mathrm{~mL}$ milk, $8 \mathrm{~g}$ oil, $25 \mathrm{~g}$ mayonnaise, and $50 \mathrm{~g}$ vegetables $(53.4 \mathrm{~g}$ fat, $30.7 \mathrm{~g}$ protein, and $50 \mathrm{~g} \mathrm{CHO}$ ) and an isocaloric LFM consisting of $312 \mathrm{~g}$ rice, $100 \mathrm{~g}$ vegetable soup, $200 \mathrm{~g}$ vegetables, $190 \mathrm{~mL}$ orange juice, $400 \mathrm{~g}$ apple, and $50 \mathrm{~g}$ kimchi $(3 \mathrm{~g}$ fat, $15.7 \mathrm{~g}$ protein, and $178 \mathrm{~g} \mathrm{CHO})$. Authors found decreased postprandial TG and oxidative burst in CAD patients [50], but no effect was observed on TG and malondialdehyde (MDA) in healthy subjects [51]. The different effects could most likely be related to both the healthy status and the different fat content of the meals rather than to the presence of fruit and fruit juice in the LFM.

Only Ghanim et al. [20] investigated the effect of orange juice on the inflammatory markers (Table 2). When a HFHCM (egg-muffin and sausage-muffin sandwiches and 2 hash-brown potatoes) was ingested with water or glucose, there was a significant rise in TLR2 or TLR 4 mRNA or protein, as well as of the plasma concentration of endotoxin and oxidative burst. All these effects were inhibited by orange juice (Table 2 ).

Overall, the results from the studies that have investigated the effect of fruit juice on postprandial stress are scarce and contrasting in drawing any firm conclusion.

\section{Conclusion}

Conflicting results have been reported on the effect of FBD on postprandial stress (Tables 1 and 2). Overall, the results suggest a complex relationship between postprandial dysmetabolism, inflammation, and oxidative stress.

Unfortunately, lipids-challenge has not been standardised and the results cannot be compared very well between studies (Figure 1). Furthermore, the selection of an appropriate control group is a critically important aspect when designing human studies. The purpose of the study dictates the choice of the appropriate placebo. In particular, studies that aim to evaluate the effects of the antioxidants contained in fruit juices should have a placebo identical not only in the total caloric and sugar content but also in the different proportion of glucose and fructose (Figure 1). Only two studies reported the use of a placebo that respects this feature (Table 1). On the other hand, studies that aim to evaluate the effects of FBD as a whole should use water as control, in order to evaluate the effect of both beneficial (antioxidants and fiber) and detrimental (sugars and fructose in particular) components of fruit juice (Figure 1). Also in this case only few studies (Table 2 ) are available and suggest that orange juice could increase postprandial insulin response. Accordingly, although results from meta-analysis reported that fruit juices did not significantly affect the concentrations of fasting glucose and insulin, they significantly increased the homeostatic model assessment insulin resistance index (HOMA-IR) values [52]. However, while a higher intake of sugar-sweetened fruit juice was significantly associated with a greater risk of $\mathrm{T} 2 \mathrm{D}$, there was no association between the intake of $100 \%$ fruit juice and the risk of T2D [53], suggesting that the added sugar was the cause of the increased risk. On the other hand, the high fructose content of the Western-style diet, along with its high fat content, may also be responsible for endotoxemia originating from the gut [54].

Concerning the inflammatory and oxidative stress markers, both markers need further analytical validation and normal ranges should be established in order to draw any firm conclusion. Therefore, caution should be taken in the interpretation of the effect of FBD in postprandial studies and the reviewed results suggest that dietary recommendations should aim to limit rather than increase sugar-sweetened beverages consumption.

\section{Abbreviations}

ATP: Atherosclerosis-prone phenotype

CAD: $\quad$ Coronary artery disease

CHO: Carbohydrates

CVD: $\quad$ Cardiovascular disease

DL: Dyslipidaemic

DUR: $\quad$ During

FBD: $\quad$ Fruit-based drink

FRAP: $\quad$ Ferric reducing antioxidant potential

HCM: $\quad$ High carbohydrates meal

HFHCM: High fat and high carbohydrates meal

HFM: High fat meal

HOMA-IR: Homeostatic model assessment insulin resistance index

IBD: Inflammatory bowel disease

IBS: $\quad$ Irritable bowel syndrome

IL: Interleukin

LFM: $\quad$ Low-fat meal

LPS: $\quad$ Lipopolysaccharide

MDA: Malondialdehyde

NAFLD: Nonalcoholic fatty liver disease

NEAC: Nonenzymatic antioxidant capacity

OW: Overweight

oxLDL: Oxidized low density lipoproteins

PRE: $\quad$ Before

ROS: $\quad$ Reactive oxygen species

T2D: $\quad$ Type 2 diabetes

TC: Total cholesterol

TG: $\quad$ Triglycerides

TLR: Toll-like receptor

TNF: Tumor necrosis factor

TRAP: Total radical-trapping antioxidant parameter

UA: $\quad$ Uric acid. 


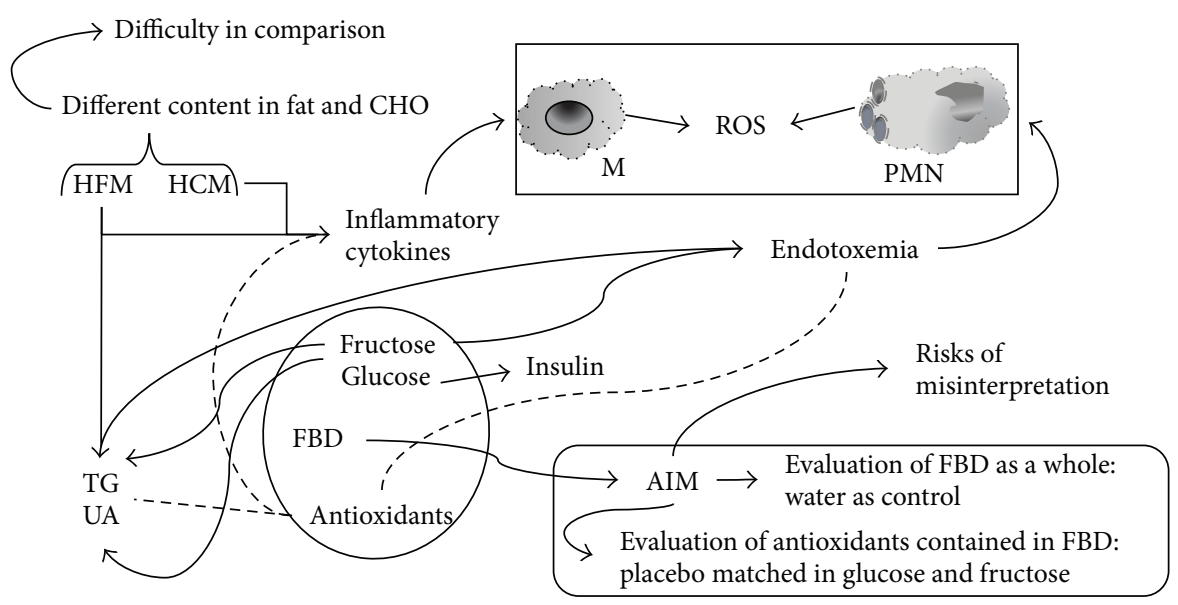

FIGURE 1: Difficulty in comparison of postprandial studies and risk of misinterpretation due to control choice. CHO: carbohydrates; FBD: fruit-based drink; HCM: high carbohydrates meal; HFM: high fat meal; M: monocytes, PMN: polymorphonuclear cells; ROS: reactive oxygen species; TG: triglycerides; UA: uric acid.

\section{Conflict of Interests}

The authors declare that there is no conflict of interests regarding the publication of this paper.

\section{Acknowledgment}

The authors thank Claudio Andrew Gobbi for English revision of the paper.

\section{References}

[1] P. C. Calder, N. Ahluwalia, R. Albers et al., "A consideration of biomarkers to be used for evaluation of inflammation in human nutritional studies," British Journal of Nutrition, vol. 109, supplement 1, pp. S1-S34, 2013.

[2] B. Burton-Freeman, "Postprandial metabolic events and fruitderived phenolics: a review of the science," British Journal of Nutrition, vol. 104, no. 3, pp. S1-S14, 2010.

[3] G. Morabito, C. Miglio, I. Peluso, and M. Serafini, "Fruit polyphenols and postprandial inflammatory stress," in Polyphenols in Human Health and Disease, vol. 2, pp. 1107-1126, Elsevier, New York, NY, USA, 2013.

[4] I. Peluso, H. Manafikhi, and M. Palmery, "Free radicals generated by post-prandial oxidative burst in the early alterations of vascular contractility," Clinical Immunology, Endocrine \& Metabolic Drugs, vol. 1, no. 1, pp. 27-45, 2014.

[5] D. Lettieri-Barbato, F. Tomei, A. Sancini, G. Morabito, and M. Serafini, "Effect of plant foods and beverages on plasma non-enzymatic antioxidant capacity in human subjects: a metaanalysis," British Journal of Nutrition, vol. 109, no. 9, pp. 15441556, 2013.

[6] I. Peluso, C. Miglio, G. Morabito, F. Ioannone, and M. Serafini, "Flavonoids and immune function in human: a systematic review," Critical Reviews in Food Science and Nutrition, vol. 55, no. 3, pp. 383-395, 2015.

[7] S. Zelber-Sagi, V. Ratziu, and R. Oren, "Nutrition and physical activity in NAFLD: an overview of the epidemiological evidence," World Journal of Gastroenterology, vol. 17, no. 29, pp. 3377-3389, 2011.
[8] R. P. MacDermott, "Treatment of irritable bowel syndrome in outpatients with inflammatory bowel disease using a food and beverage intolerance, food and beverage avoidance diet," Inflammatory Bowel Diseases, vol. 13, no. 1, pp. 91-96, 2007.

[9] B. Burton-Freeman, A. Linares, D. Hyson, and T. Kappagoda, "Strawberry modulates LDL oxidation and postprandial lipemia in response to high-fat meal in overweight hyperlipidemic men and women," The Journal of the American College of Nutrition, vol. 29, no. 1, pp. 46-54, 2010.

[10] I. Edirisinghe, K. Banaszewski, J. Cappozzo et al., "Strawberry anthocyanin and its association with postprandial inflammation and insulin," British Journal of Nutrition, vol. 106, no. 6, pp. 913-922, 2011.

[11] C. L. Ellis, I. Edirisinghe, T. Kappagoda, and B. BurtonFreeman, "Attenuation of meal-induced inflammatory and thrombotic responses in overweight men and women after 6-week daily strawberry (Fragaria) intake-a randomized placebo-controlled trial," Journal of Atherosclerosis and Thrombosis, vol. 18, no. 4, pp. 318-327, 2011.

[12] P. Huebbe, K. Giller, S. de Pascual-Teresa et al., "Effects of blackcurrant-based juice on atherosclerosis-related biomarkers in cultured macrophages and in human subjects after consumption of a high-energy meal," British Journal of Nutrition, vol. 108, no. 2, pp. 234-244, 2012.

[13] C. D. Kay and B. J. Holub, “The effect of wild blueberry (Vaccinium angustifolium) consumption on postprandial serum antioxidant status in human subjects," British Journal of Nutrition, vol. 88, no. 4, pp. 389-398, 2002.

[14] A. S. Mathew, G. M. Capel-Williams, S. E. E. Berry, and W. L. Hall, "Acute effects of pomegranate extract on postprandial lipaemia, vascular function and blood pressure," Plant Foods for Human Nutrition, vol. 67, no. 4, pp. 351-357, 2012.

[15] G. Mazza, C. D. Kay, T. Cottrell, and B. J. Holub, "Absorption of anthocyanins from blueberries and serum antioxidant status in human subjects," Journal of Agricultural and Food Chemistry, vol. 50, no. 26, pp. 7731-7737, 2002.

[16] C. Miglio, I. Peluso, A. Raguzzini et al., "Fruit juice drinks prevent endogenous antioxidant response to high-fat meal ingestion," British Journal of Nutrition, vol. 111, no. 2, pp. 294300, 2014. 
[17] I. Peluso, A. Raguzzini, D. V. Villano et al., "High fat meal increase of IL-17 is prevented by ingestion of fruit juice drink in healthy overweight subjects," Current Pharmaceutical Design, vol. 18, no. 1, pp. 85-90, 2012.

[18] I. Peluso, D. V. Villano, S. A. Roberts et al., "Consumption of mixed fruit-juice drink and vitamin $\mathrm{C}$ reduces postprandial stress induced by a high fat meal in healthy overweight subjects," Current Pharmaceutical Design, vol. 20, no. 6, pp. 1020-1024, 2014.

[19] S. M. Snyder, J. D. Reber, B. L. Freeman, K. Orgad, D. L. Eggett, and T. L. Parker, "Controlling for sugar and ascorbic acid, a mixture of flavonoids matching navel oranges significantly increases human postprandial serum antioxidant capacity," Nutrition Research, vol. 31, no. 7, pp. 519-526, 2011.

[20] H. Ghanim, C. L. Sia, M. Upadhyay et al., "Orange juice neutralizes the proinflammatory effect of a high-fat, highcarbohydrate meal and prevents endotoxin increase and tolllike receptor expression," The American Journal of Clinical Nutrition, vol. 91, no. 4, pp. 940-949, 2010.

[21] S. M. Hampton, C. Isherwood, V. J. E. Kirkpatrick, A. C. LynneSmith, and B. A. Griffin, "The influence of alcohol consumed with a meal on endothelial function in healthy individuals," Journal of Human Nutrition and Dietetics, vol. 23, no. 2, pp. 120$125,2010$.

[22] S. Panahi, D. El Khoury, B. L. Luhovyy, H. D. Goff, and G. H. Anderson, "Caloric beverages consumed freely at meal-time add calories to an ad libitum meal," Appetite, vol. 65, pp. 75-82, 2013.

[23] J. D. Stookey, J. Hamer, G. Espinoza et al., “Orange juice limits postprandial fat oxidation after breakfast in normal-weight adolescents and adults," Advances in Nutrition, vol. 3, no. 4, pp. 629S-635S, 2012.

[24] C. G. McCarthy, T. M. Farney, R. E. Canale, M. E. Dessoulavy, and R. J. Bloomer, "High-fat feeding, but not strenuous exercise, increases blood oxidative stress in trained men," Applied Physiology, Nutrition and Metabolism, vol. 38, no. 1, pp. 33-41, 2013.

[25] V. Englert, K. Wells, W. Long, M. S. Hickey, and C. L. Melby, "Effect of acute prior exercise on glycemic and insulinemic indices," Journal of the American College of Nutrition, vol. 25, no. 3, pp. 195-202, 2006.

[26] M. C. Peddie, J. L. Bone, N. J. Rehrer, C. M. Skeaff, A. R. Gray, and T. L. Perry, "Breaking prolonged sitting reduces postprandial glycemia in healthy, normal-weight adults: a randomized crossover trial," The American Journal of Clinical Nutrition, vol. 98, no. 2, pp. 358-366, 2013.

[27] K. L. Timmerman, M. G. Flynn, P. M. Coen, M. M. Markofski, and B. D. Pence, "Exercise training-induced lowering of inflammatory $(\mathrm{CD} 14+\mathrm{CD} 16+)$ monocytes: a role in the antiinflammatory influence of exercise?" Journal of Leukocyte Biology, vol. 84, no. 5, pp. 1271-1278, 2008.

[28] N. T. Jenkins, R. Q. Landers, S. R. Thakkar et al., "Prior endurance exercise prevents postprandial lipaemia-induced increases in reactive oxygen species in circulating CD $31^{+}$cells," Journal of Physiology, vol. 589, no. 22, pp. 5539-5553, 2011.

[29] C. E. Melton, P. S. Tucker, K. H. Fisher-Wellman, B. K. Schilling, and R. J. Bloomer, "Acute exercise does not attenuate postprandial oxidative stress in prediabetic women," Physician and Sportsmedicine, vol. 37, no. 1, pp. 27-36, 2009.

[30] M. Kumawat, T. K. Sharma, N. Singh et al., "Study of changes in antioxidant enzymes status in diabetic post menopausal group of women suffering from cardiovascular complications," Clinical Laboratory, vol. 58, no. 3-4, pp. 203-207, 2012.
[31] S. Neri, S. Calvagno, B. Mauceri et al., "Effects of antioxidants on postprandial oxidative stress and endothelial dysfunction in subjects with impaired glucose tolerance and type 2 diabetes," European Journal of Nutrition, vol. 49, no. 7, pp. 409-416, 2010.

[32] F. Laugerette, C. Vors, N. Peretti, and M.-C. Michalski, "Complex links between dietary lipids, endogenous endotoxins and metabolic inflammation," Biochimie, vol. 93, no. 1, pp. 39-45, 2011.

[33] R. Deopurkar, H. Ghanim, J. Friedman et al., "Differential effects of cream, glucose, and orange juice on inflammation, endotoxin, and the expression of toll-like receptor-4 and suppressor of cytokine signaling-3," Diabetes Care, vol. 33, no. 5, pp. 991-997, 2010.

[34] S. B. Lotito and B. Frei, "The increase in human plasma antioxidant capacity after apple consumption is due to the metabolic effect of fructose on urate, not apple-derived antioxidant flavonoids," Free Radical Biology and Medicine, vol. 37, no. 2, pp. 251-258, 2004.

[35] K. L. Teff, S. S. Elliott, M. Tschöp et al., "Dietary fructose reduces circulating insulin and leptin, attenuates postprandial suppression of ghrelin, and increases triglycerides in women," The Journal of Clinical Endocrinology \& Metabolism, vol. 89, no. 6, pp. 2963-2972, 2004.

[36] K. L. Teff, J. Grudziak, R. R. Townsend et al., "Endocrine and metabolic effects of consuming fructose- and glucosesweetened beverages with meals in obese men and women: influence of insulin resistance on plasma triglyceride responses," Journal of Clinical Endocrinology and Metabolism, vol. 94, no. 5, pp. 1562-1569, 2009.

[37] K. L. Stanhope and P. J. Havel, "Endocrine and metabolic effects of consuming beverages sweetened with fructose, glucose, sucrose, or high-fructose corn syrup," The American Journal of Clinical Nutrition, vol. 88, no. 6, pp. 1733S-1737S, 2008.

[38] M. M. Swarbrick, K. L. Stanhope, S. S. Elliott et al., "Consumption of fructose-sweetened beverages for 10 weeks increases postprandial triacylglycerol and apolipoprotein-B concentrations in overweight and obese women," British Journal of Nutrition, vol. 100, no. 5, pp. 947-952, 2008.

[39] L. Tappy and K.-A. Lê, "Does fructose consumption contribute to non-alcoholic fatty liver disease?" Clinics and Research in Hepatology and Gastroenterology, vol. 36, no. 6, pp. 554-560, 2012.

[40] R. Jin, N.-A. Le, S. Liu et al., "Children with NAFLD are more sensitive to the adverse metabolic effects of fructose beverages than children without NAFLD," The Journal of Clinical Endocrinology and Metabolism, vol. 97, no. 7, pp. E1088-E1098, 2012.

[41] T. D. Heden, Y. Liu, Y.-M. Park, L. M. Nyhoff, N. C. Winn, and J. A. Kanaley, "Moderate amounts of fructose- or glucosesweetened beverages do not differentially alter metabolic health in male and female adolescents," American Journal of Clinical Nutrition, vol. 100, no. 3, pp. 796-805, 2014.

[42] A. G. Ruiz, F. Casafont, J. Crespo et al., "Lipopolysaccharidebinding protein plasma levels and liver TNF-alpha gene expression in obese patients: evidence for the potential role of endotoxin in the pathogenesis of non-alcoholic steatohepatitis," Obesity Surgery, vol. 17, no. 10, pp. 1374-1380, 2007.

[43] Y. S. Roh and E. Seki, "Toll-like receptors in alcoholic liver disease, non-alcoholic steatohepatitis and carcinogenesis," Journal of Gastroenterology and Hepatology, vol. 28, supplement 1, pp. 38-42, 2013. 
[44] D. Satoh, T. Yagi, T. Nagasaka et al., "CD14 upregulation as a distinct feature of non-alcoholic fatty liver disease after pancreatoduodenectomy," World Journal of Hepatology, vol. 5, no. 4, pp. 189-195, 2013.

[45] T. Unno, M. Tago, Y. Suzuki et al., "Effect of tea catechins on postprandial plasma lipid responses in human subjects," British Journal of Nutrition, vol. 93, no. 4, pp. 543-547, 2005.

[46] R. Törrönen, M. Kolehmainen, E. Sarkkinen, H. Mykkänen, and L. Niskanen, "Postprandial glucose, insulin, and free fatty acid responses to sucrose consumed with blackcurrants and lingonberries in healthy women," The American Journal of Clinical Nutrition, vol. 96, no. 3, pp. 527-533, 2012.

[47] C. Miglio, I. Peluso, A. Raguzzini et al., "Antioxidant and inflammatory response following high-fat meal consumption in overweight subjects," European Journal of Nutrition, vol. 52, no. 3, pp. 1107-1114, 2013.

[48] M. J. Sullivan and R. L. Scott, "Postprandial glycemic response to orange juice and nondiet cola: is there a difference?" The Diabetes Educator, vol. 17, no. 4, pp. 274-278, 1991.

[49] A. Ramel, F. D. Gudmundsdottir, and I. Thorsdottir, "Effects of two different types of fast food on postprandial metabolism in normal and overweight subjects," European Journal of Clinical Nutrition, vol. 66, no. 11, pp. 1193-1198, 2012.

[50] J.-H. Bae, E. Bassengel, H.-J. Lee et al., "Impact of postprandial hypertriglyceridemia on vascular responses in patients with coronary artery disease: effects of ACE inhibitors and fibrates," Atherosclerosis, vol. 158, no. 1, pp. 165-171, 2001.

[51] J.-H. Bae, M. Schwemmer, I.-K. Lee et al., "Postprandial hypertriglyceridemia-induced endothelial dysfunction in healthy subjects is independent of lipid oxidation," International Journal of Cardiology, vol. 87, no. 2-3, pp. 259-267, 2003.

[52] B. Wang, K. Liu, M. Mi, and J. Wang, "Effect of fruit juice on glucose control and insulin sensitivity in adults: a meta-analysis of 12 randomized controlled trials," PLoS ONE, vol. 9, no. 4, Article ID e95323, 2014.

[53] B. Xi, S. Li, Z. Liu et al., "Intake of fruit juice and incidence of type 2 diabetes: a systematic review and meta-analysis," PLoS ONE, vol. 9, no. 3, p. e93471, 2014.

[54] R. H. Lustig, "Fructose: metabolic, hedonic, and societal parallels with ethanol., Journal of the American Dietetic Association, vol. 110, no. 9, pp. 1307-1321, 2010. 


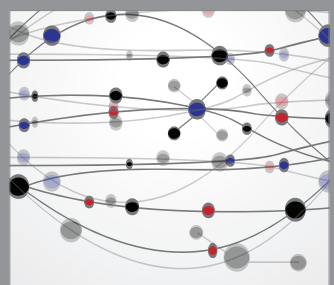

The Scientific World Journal
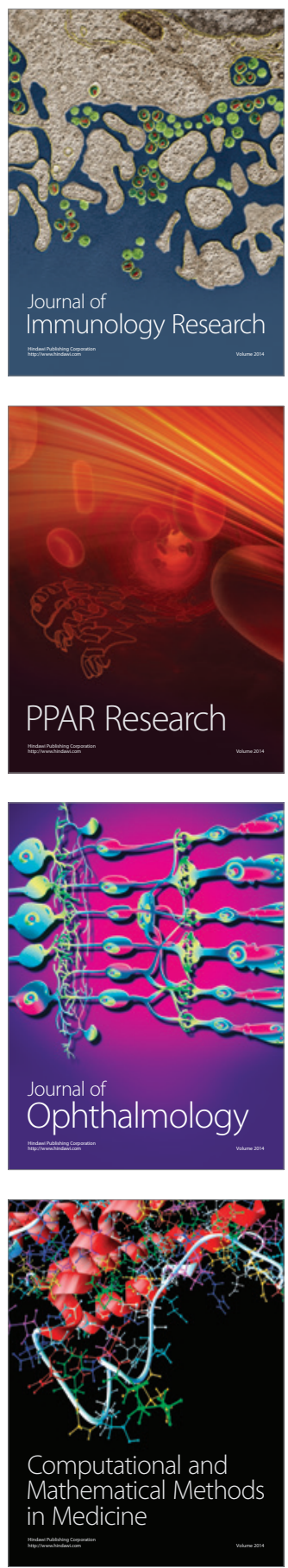

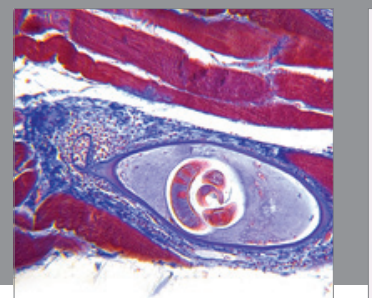

Gastroenterology

Research and Practice
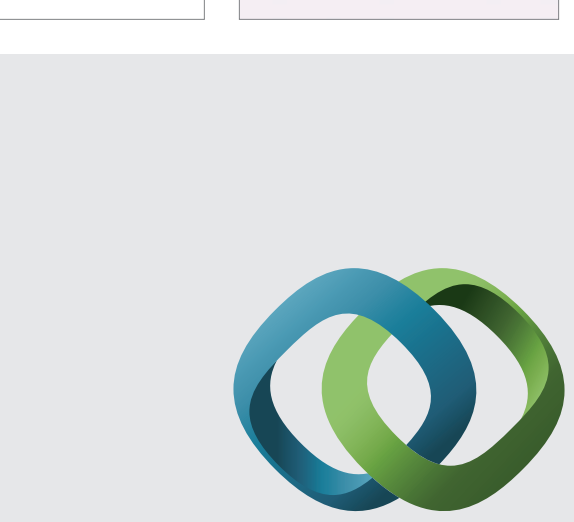

\section{Hindawi}

Submit your manuscripts at

http://www.hindawi.com
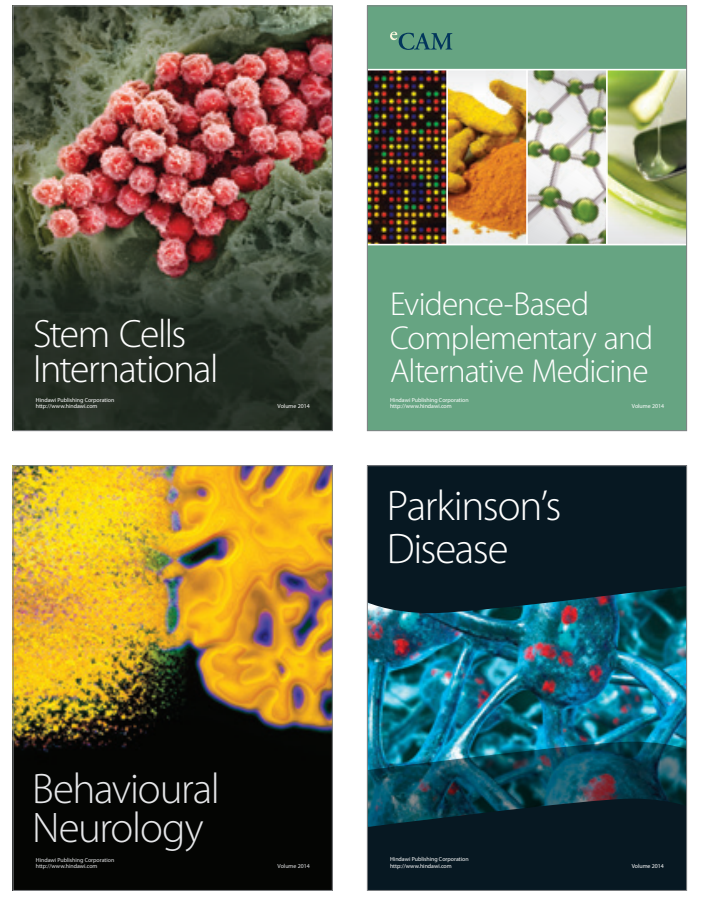
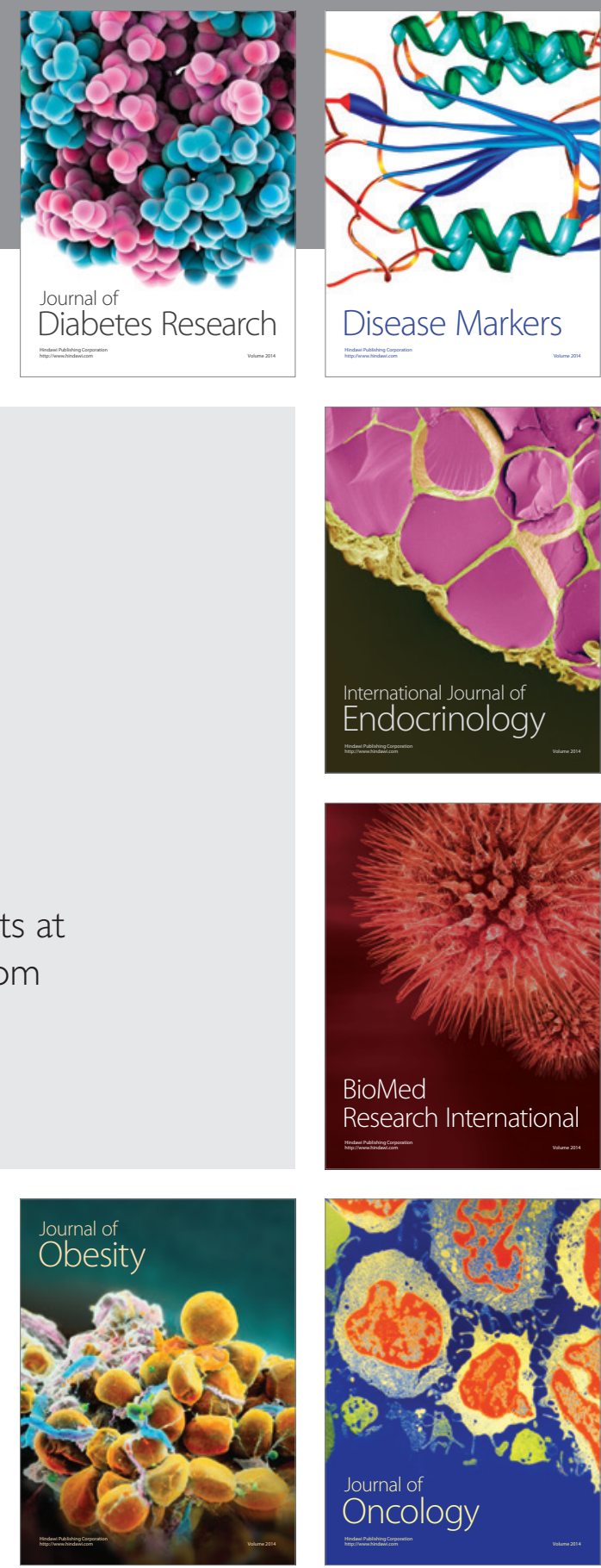

Disease Markers
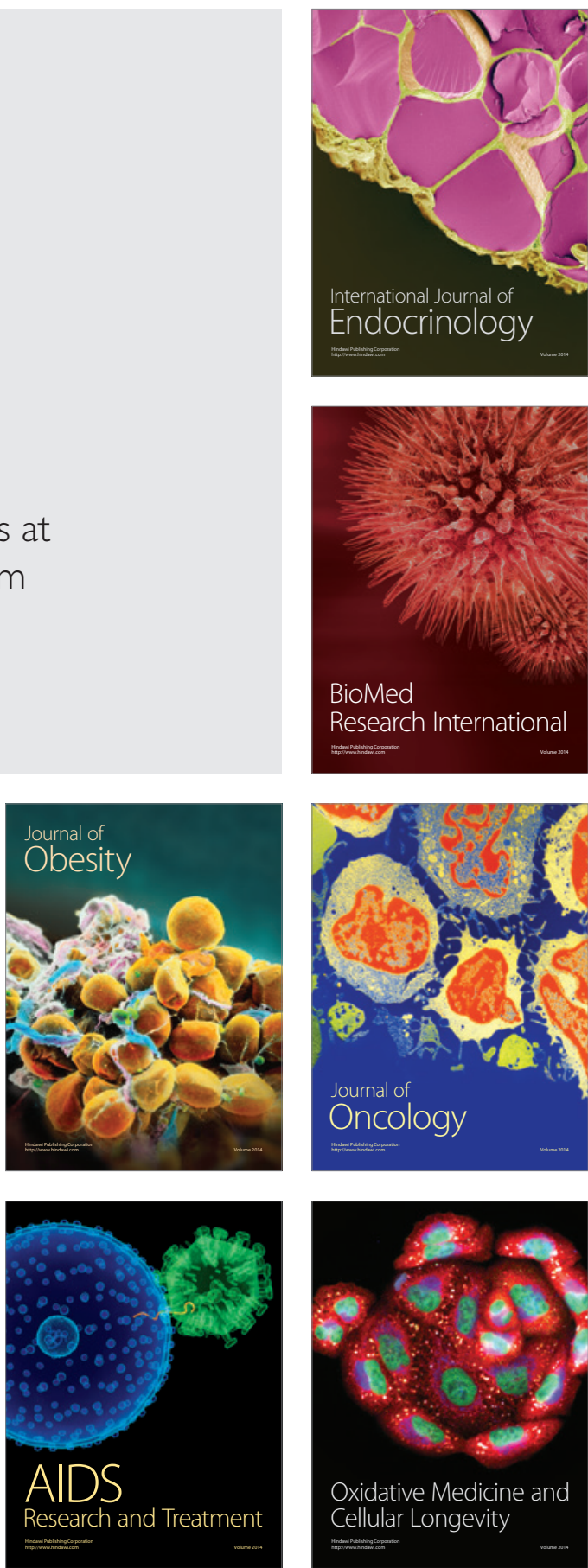